\title{
Circulatory Consequences of Changes in Cardiac Rhythm Produced in Patients by Transthoracic Direct-Current Shock*
}

\author{
John S. Graettinger, Richard A. Carleton, and Joseph J. Muenster \\ (From the Section of Cardio-respiratory Diseases, Department of Medicine, Presbyterian- \\ St. Luke's Hospital, and the Department of Medicine, University of Illinois \\ College of Medicine, Chicago, Ill.)
}

Most arrhythmias have long been regarded as undesirable. The salutary clinical consequences of abolishing ventricular and atrial tachycardias are often immediately apparent, and, in general, the circulatory disadvantages of an inappropriately rapid ventricular rate in man would seem well accepted.

The effect on the circulation of atrial arrhythmias without tachycardia, particularly atrial fibrillation, however, is not clear. Lower cardiac outputs have been reported in groups of patients with rheumatic heart disease and atrial fibrillation than in those with sinus rhythm $(1,2)$. Studies of the same patients first with atrial fibrillation and later in sinus rhythm after quinidine conversion have been reported by several investigators (3-7). Of the 35 patients in two of these studies in which only mean data are presented $(4,7)$, the majority of patients had increased outputs after conversion. Of the 27 patients in the other three studies $(3,5,6)$, the output was increased at rest after conversion in eighteen and was unchanged or less in nine. In the two studies in which heart rates were available $(4,5)$, no rates of over 100 in atrial fibrillation were found. In published data, therefore, it is evident that restoration of sinus rhythm with quinidine does not regularly result in an increased cardiac output when measurements are made days to weeks after conversion.

* Submitted for publication June 10, 1964; accepted August 7, 1964.

Supported in part by grants from the U. S. Public Health Service, National Institutes of Health (HE05579), and from the Chicago and Illinois Heart Associations.

Presented in part at the 55th annual meeting of the American Society for Clinical Investigation, Atlantic City, N. J., April 29, 1963.
Since quinidine was used as the antiarrhythmic drug in all of these studies, the observations on circulatory function were necessarily made days to weeks after the instant of reversion, i.e., at a time when over-all circulatory adjustments to the rhythm change would be anticipated. The technique of direct-current transthoracic shock, timed to occur during maximal ventricular depolarization, has been shown by Lown, Neuman, Amarasingham, and Berkovits in animals and man $(8,9)$ to be safe and effective in terminating various arrhythmias; furthermore, it has made possible hemodynamic studies predictably timed to coincide with a change in cardiac rhythm in the absence of quinidine. We have made observations before, during, and after condenser-discharge shocks in 26 patients with various arrhythmias undergoing electrical conversion attempts. The majority of these patients had atrial fibrillation with varying rates of ventricular response; 21 detailed hemodynamic studies were made in 20 of these patients in an effort to assess the relative importance of the ventricular rate and the absence of atrial systole on cardiac output.

\section{Methods}

The patients studied included four with ischemic, 16 with rheumatic, and two with hypertensive heart disease; one patient had had an atrial septal defect closed, and three patients, one with borderline hyperthyroidism [BMR, +20\% ; PBI (protein-bound iodine), $9.2 \mu \mathrm{g}$ per $100 \mathrm{ml}$ ] and one with labile blood pressure, had atrial fibrillation as the only sign we could detect of possible heart disease (Table I). In 13, cardiac surgery had been performed. Each patient was receiving a digitalis preparation and was considered to be "digitalized" on the evening before study. Quinidine had been given to each in varying amounts before the conversion attempt, but no patient had received quinidine within the 24 hours before study. All patients had cardiac enlargement; the 
TABLE I

Clinical data of patients undergoing transthoracic shock*

\begin{tabular}{|c|c|c|c|c|c|c|c|c|c|c|c|}
\hline \multirow[b]{2}{*}{ Patient } & \multirow[b]{2}{*}{$\begin{array}{l}\text { Age } \\
\text { Sex }\end{array}$} & \multicolumn{6}{|c|}{ Cardiac status } & \multicolumn{4}{|c|}{ Result of shock } \\
\hline & & Heart disease & Class & Arrhythmia & $\begin{array}{l}\text { Dur } \\
\text { arrh }\end{array}$ & $\begin{array}{l}\text { ation of } \\
\text { ythmia }\end{array}$ & $\begin{array}{l}\text { Cardio- } \\
\text { thoracic } \\
\text { ratio }\end{array}$ & Rhythm & Dur & $\begin{array}{l}\text { tion of } \\
\text { SR }\end{array}$ & Class \\
\hline 1a. H.M. & $58 \mathrm{M}$ & Ischemic & 2 & A.Fl. & 3 & mos & 54.0 & $\mathrm{NSR} \rightarrow$ A.Fl. & 48 & hrs & 2 \\
\hline b. H.M. & $58 \mathrm{M}$ & Ischemic & 2 & A.Fl. & 48 & hrs & 54.0 & $\mathrm{NSR} \rightarrow$ A.Fl. & 48 & hrs & 2 \\
\hline 2. F.S. & $78 \mathrm{M}$ & Ischemic & 2 & A.Fi. & 1 & mo & 49.5 & NSR & 13 & mos & 1 \\
\hline 3. L.D. & $42 \mathrm{M}$ & Ischemic & 4 & A.Fl. & 7 & days & 53.5 & NSR & 9 & $\operatorname{mos}$ & 2 \\
\hline 4. C.L. & $55 \mathrm{M}$ & Ischemic & 4 & V.Tach. & 4 & days & & NSR & 6 & mos & 1 \\
\hline 5. S.T. & $42 \mathrm{~F}$ & RHD-MS $\dagger$ & 1 & AF & 7 & days & 55.0 & NSR & 13 & mos & 1 \\
\hline 6. M.S. & $54 \mathrm{~F}$ & RHD-M I & 2 & $\mathrm{AF}$ & 17 & mos & 54.0 & $\mathrm{NSR} \rightarrow \mathrm{AF}$ & $2 \frac{1}{2}$ & $\operatorname{mos}$ & 1 \\
\hline 7a. J.P. & $52 \mathrm{M}$ & RHD-MS† & 2 & $\mathrm{AF}$ & 2 & mos & 53.5 & $\mathrm{NSR} \rightarrow \mathrm{AF}$ & 8 & days & 2 \\
\hline b. J.P. & $52 \mathrm{M}$ & RHD-MS & 2 & $\mathrm{AF}$ & 40 & days & 53.5 & $\mathrm{NSR} \rightarrow \mathrm{AF}$ & 13 & days & 2 \\
\hline 8. G.S. & $40 \mathrm{~F}$ & RHD-MS\$ & 2 & $\mathrm{AF}$ & 34 & mos & 52.0 & NSR & 12 & $\operatorname{mos}$ & 1 \\
\hline 9a. A.L. & $46 \mathrm{~F}$ & RHD-MSt & 1 & $\mathrm{AF}$ & 2 & mos & 58.0 & $\mathrm{NSR} \rightarrow \mathrm{AF}$ & 8 & days & 1 \\
\hline b. A.L. & $46 \mathrm{~F}$ & RHD-MSt & 1 & $\mathrm{AF}$ & 40 & days & 55.0 & $\mathrm{NSR} \rightarrow \mathrm{AF}$ & 8 & days & 1 \\
\hline 10. R.P. & $53 \mathrm{~F}$ & RHD-MS-TI & 2 & $\mathrm{AF}$ & 4 & yrs & 53.0 & $\mathrm{NSR} \rightarrow \mathrm{AF}$ & 10 & days & 2 \\
\hline 11. A.C. & $45 \mathrm{M}$ & RHD-MS & 1 & $\mathrm{AF}$ & 9 & days & 54.5 & NSR & $8 \frac{1}{2}$ & mos & 1 \\
\hline 12. E.T. & $55 \mathrm{~F}$ & RHD-MS $\ddagger$ & 1 & $\mathrm{AF}$ & 5 & yrs & 65.0 & NSR & 8 & mos & 1 \\
\hline 13. C.B. & $40 \mathrm{~F}$ & RHD-MS $\S$ & 2 & $\mathrm{AF}$ & 1 & $\mathrm{yr}$ & 56.0 & NSR & 8 & mos & 1 \\
\hline 14. L.A. & $33 \mathrm{~F}$ & RHD-MS $\$$ & 2 & $\mathrm{AF}$ & 2 & yrs & 62.0 & $\mathrm{NSR} \rightarrow \mathrm{AF}$ & 14 & days & 2 \\
\hline 15. C.P. & $48 \mathrm{~F}$ & RHD-MS & 1 & $\mathrm{AF}$ & 5 & yrs & 56.0 & NSR & 6 & mos & 1 \\
\hline 16. M.I. & $46 \mathrm{~F}$ & RHD-MI & 1 & $\mathrm{AF}$ & 9 & mos & 55.5 & $\mathrm{AF}$ & & & 1 \\
\hline 17. B.D. & $43 \mathrm{~F}$ & RHD-MS $\$$ & 2 & $\mathrm{AF}$ & 15 & yrs & 66.0 & $\mathrm{AF}$ & & & 2 \\
\hline 18. M.H. & $40 \mathrm{~F}$ & RHD-MS§ & 2 & $\mathrm{AF}$ & 7 & yrs & 65.5 & $\mathrm{AF}$ & & & 2 \\
\hline 19. L.S. & $40 \mathrm{~F}$ & RHD-MI $\$$ & 2 & $\mathrm{AF}$ & 7 & yrs & 58.0 & $\mathrm{AF}$ & & & 2 \\
\hline 20. M.C. & $40 \mathrm{~F}$ & RHD-MI & 2 & $\mathrm{AF}$ & 4 & mos & 58.5 & NSR & 6 & $\operatorname{mos}$ & 2 \\
\hline 21. P.L. & $40 \mathrm{M}$ & $\mathrm{HHD}^{+}$ & 2 & A.F1. & 9 & mos & 49.5 & NSR & 12 & mos & 1 \\
\hline 22. A.A. & $64 \mathrm{M}$ & HHD & 2 & $\mathrm{AF}$ & 2 & yrs & 54.5 & $\mathrm{NSR} \rightarrow \mathrm{AF}$ & 4 & days & 2 \\
\hline 23. A.E. & $53 \mathrm{~F}$ & ASD $\S$ & 1 & $\mathrm{AF}$ & 5 & yrs & 69.5 & NSR & 8 & mos & 1 \\
\hline 24. C.S. & $58 \mathrm{M}$ & Hyperthyroid? & 2 & $\mathrm{AF}$ & 10 & mos & 53.0 & $\mathrm{AF}$ & & & 2 \\
\hline 25a. W.F. & $42 \mathrm{M}$ & ? & 1 & $\mathrm{AF}$ & 6 & yrs & 61.0 & $\mathrm{NSR} \rightarrow \mathrm{AF}$ & 1 & day & 1 \\
\hline b. W.F. & $42 \mathrm{M}$ & ? & 1 & $\mathrm{AF}$ & 14 & days & 61.0 & $\mathrm{NSR} \rightarrow \mathrm{AF}$ & 30 & days & 1 \\
\hline 26. H.B. & $64 \mathrm{~F}$ & ? & 2 & $\mathrm{AF}$ & 3 & mos & 55.5 & $\mathrm{NSR} \rightarrow \mathrm{AF}$ & 5 & days & 1 \\
\hline
\end{tabular}

* Abbreviations: RHD = rheumatic heart disease; $\mathrm{MS}=$ mitral stenosis; $\mathrm{MI}=$ mitral insufficiency; TI $=$ tricuspid insufficiency; $\mathrm{HHD}=$ hypertensive heart disease; $\mathrm{ASD}=$ atrial septal defect. A.Fl. $=$ atrial flutter; V.Tach. $=$ ventricular tachycardia $; \mathrm{AF}=$ atrial fibrillation; $\mathrm{NSR}=$ normal sinus rhythm.

$\dagger$ Surgery, 9-12 days before.

$\ddagger$ Surgery, 1-4 months before.

§ Surgery, 1-15 years before.

cardiothoracic ratios calculated from 2-meter roentgenograms are shown in Table I. In 20 patients 21 hemodynamic studies were performed, by methods previously described $(10)$, in the postabsorptive state 1 to 3 hours after a sedative dose of sodium 5-allyl-5-(1 methylbutyl) barbiturate and 75 to $100 \mathrm{mg}$ of meperidine intramuscularly. After measurements of pressures and oxygen saturations during venous catheterization, direct Fick cardiac outputs were determined at rest and during the fourth minute of exercise with the patient supine on a stationary bicycle. Consecutively with the Fick outputs, the cardiac output was also estimated by an indicator-dilution technique employing indocyanine green and continuous arterial sampling. After these initial measurements the patient was lightly anesthetized with sodium thiopental intravenously, and the indicator-dilution output was repeated. The condenser-discharge shock was then given between one paddle placed over the fourth intercostal space to the right of the sternum and another in the posterior axilla just below the level of the cardiac apex. The initial shock was usually of 100-watt-seconds energy; if necessary, additional shocks up to 400 watt-seconds were given. Continuous pressure and electrocardiographic recordings were made. Immediately after the $\operatorname{shock}(\mathrm{s})$, an indicator-dilution output was repeated. The patient was then allowed to awaken from anesthesia, and 1 or 1 and 2 hours after awakening, Fick and dilution outputs were repeated at rest and during exercise. In three patients of the series, outputs were also determined at greater intervals after conversion.

In the latter patients of the series an arterial catheter was positioned in the aortic root, and another catheter was passed transeptally into the left atrium. The aortic root catheter was advanced into the left ventricle and the presence or absence of mitral regurgitation qualitatively assessed by the detection of or failure to detect indicator dye in the left atrium after left ventricular injection. The left ventricular mean-diastolic and enddiastolic pressures measured were those nearest in time to the output measurements.

All pressures were recorded with Statham P-23Db pressure transducers as part of a bridge circuit, activated by $12-\mathrm{v}$ direct current, and Heiland type $40-350 \mathrm{C}$ gal- 
TABLE II

Hemodynamic changes accompanying

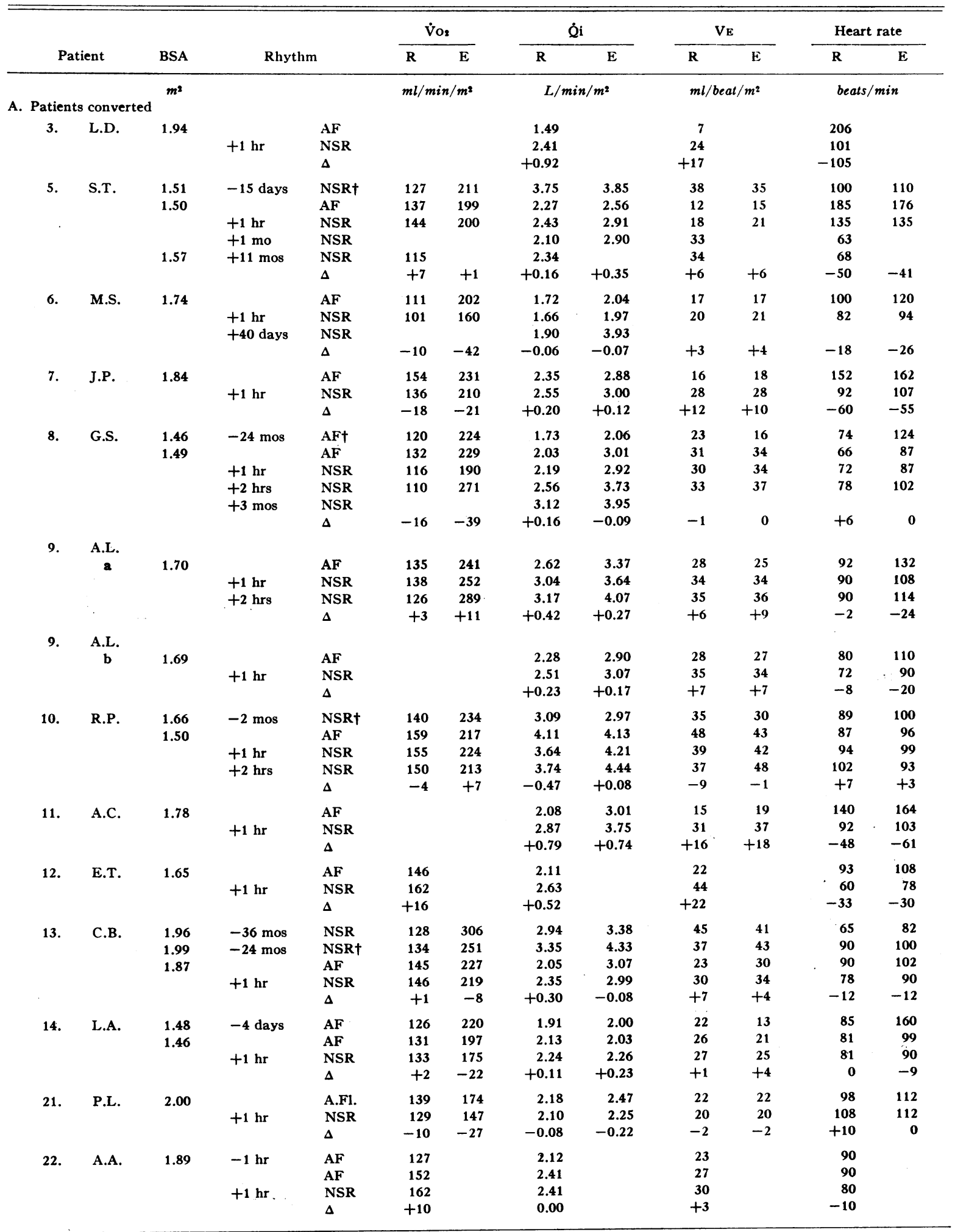


TABLE II

transthoracic shock*

\begin{tabular}{|c|c|c|c|c|c|c|c|c|c|c|c|c|c|}
\hline \multicolumn{2}{|c|}{ a-v difference } & \multicolumn{2}{|c|}{$\overline{P R A}$} & \multicolumn{2}{|c|}{$\mathbf{P} \overline{\mathbf{P A}}$} & \multicolumn{2}{|c|}{$\mathbf{P} \overline{\mathbf{B A}}$} & \multicolumn{2}{|c|}{ Pwedge } & \multicolumn{2}{|c|}{ Rs } & \multicolumn{2}{|c|}{ RPart. } \\
\hline $\mathbf{R}$ & $\mathrm{E}$ & $\mathbf{R}$ & $\mathrm{E}$ & $\mathbf{R}$ & $\mathbf{E}$ & $\mathbf{R}$ & $\mathrm{E}$ & $\mathbf{R}$ & $\mathrm{E}$ & $\mathbf{R}$ & $\mathrm{E}$ & $\mathbf{R}$ & $\mathbf{E}$ \\
\hline \multicolumn{2}{|c|}{$\mathrm{ml} / 100 \mathrm{ml}$} & \multicolumn{2}{|c|}{$m m \mathrm{Hg}$} & \multicolumn{2}{|c|}{$m m H_{g}$} & \multicolumn{2}{|c|}{$m m \mathrm{Hg}$} & \multicolumn{2}{|c|}{$m m \mathrm{Hg}$} & \multicolumn{2}{|c|}{ dyne-sec-cm ${ }^{-b}$} & \multicolumn{2}{|c|}{ dyne-sec- $\mathrm{cm}^{-b}$} \\
\hline & & & & & & $\begin{array}{r}89 \\
84 \\
-5\end{array}$ & & & & $\begin{array}{r}2,500 \\
1,400 \\
-1,100\end{array}$ & & & \\
\hline 3.39 & 5.47 & 2 & 3 & 41 & 37 & 97 & 88 & 18 & 26 & 1,400 & 1,200 & 300 & 200 \\
\hline 6.04 & 7.75 & 8 & 8 & 29 & 36 & 95 & 89 & 16 & & 2,200 & 1,900 & 300 & \\
\hline 5.90 & 6.88 & 5 & 7 & 24 & 40 & 104 & 102 & 9 & 13 & 2,300 & 1,900 & 300 & 500 \\
\hline 4.76 & & 4 & & 19 & & 88 & & 11 & & 1,900 & & 200 & \\
\hline-0.14 & -0.87 & -3 & -1 & -5 & +4 & +9 & +13 & -7 & & +100 & $\mathbf{0}$ & 0 & \\
\hline 6.46 & 9.90 & 7 & 8 & 28 & 39 & 67 & 63 & 18 & & 1,800 & 1,400 & & \\
\hline 6.10 & 8.10 & 6 & 7 & 29 & 35 & 64 & 73 & & & 1,800 & 1,700 & & \\
\hline-0.36 & -1.80 & -1 & -1 & +1 & -4 & -3 & +10 & & & 0 & +300 & & \\
\hline 6.56 & 8.04 & 10 & 11 & 40 & 54 & 74 & 93 & & & 1,400 & 1,400 & & \\
\hline 5.32 & 7.00 & 6 & 10 & 44 & 58 & 86 & 95 & & & 1,500 & 1.400 & & \\
\hline-1.24 & -1.04 & -4 & -1 & +4 & +4 & +12 & +2 & & & +100 & 0 & & \\
\hline 6.92 & 10.88 & 6 & 6 & 25 & 35 & 85 & 86 & 21 & 25 & 2,700 & 2,300 & 100 & 300 \\
\hline 6.51 & 7.62 & 7 & & 19 & 25 & 95 & 84 & 16 & 17 & 2,500 & 1,500 & 100 & 100 \\
\hline 5.26 & 6.51 & & & 21 & 24 & 90 & 94 & & & 2,200 & 1,700 & & \\
\hline 4.27 & 7.29 & & & 20 & 27 & 101 & 108 & & & 2,100 & 1,600 & & \\
\hline-1.25 & -1.11 & & & +2 & -1 & -5 & +10 & & & -300 & +200 & & \\
\hline 5.15 & 7.15 & 10 & & 26 & 36 & 93 & 106 & 19 & & 1,700 & 1,500 & 100 & \\
\hline 4.52 & 6.93 & & & 25 & 44 & 80 & 105 & & & 1,200 & 1,400 & & \\
\hline 3.97 & 7.10 & & 13 & 28 & 44 & 79 & 103 & & & 1,200 & 1,200 & & \\
\hline \multirow{4}{*}{-0.63} & -0.22 & & & -1 & +8 & -13 & -1 & & & -500 & -100 & & \\
\hline & & & & & & 88 & 95 & & & 1,800 & 1,600 & & \\
\hline & & & & & & 88 & 93 & & & 1.700 & 1,400 & & \\
\hline & & & & & & 0 & -2 & & & -100 & -200 & & \\
\hline 4.54 & 7.87 & 19 & & 35 & 42 & 89 & 95 & 24 & 30 & 1,400 & 1,500 & 200 & 200 \\
\hline 3.86 & 5.24 & 11 & & 21 & 26 & 96 & 102 & 12 & & 1,200 & 1,300 & 100 & \\
\hline 4.27 & 5.32 & & & 21 & 24 & 94 & 95 & & & 1,400 & 1,200 & & \\
\hline 4.01 & 4.79 & & & 22 & 22 & 92 & 88 & 18 & & 1.300 & 1,100 & 100 & \\
\hline+0.41 & +0.08 & & & 0 & -2 & -2 & -7 & & & +200 & -100 & & \\
\hline & & & & & & 74 & 70 & & & 1,600 & 1,000 & & \\
\hline & & & & & & 74 & 84 & & & 1,200 & 1,000 & & \\
\hline & & & & & & 0 & +14 & & & -400 & 0 & & \\
\hline 6.91 & 8.54 & 7 & & 29 & 33 & 105 & 103 & 15 & 17 & 2,400 & & 300 & \\
\hline 6.15 & 7.20 & & 8 & 33 & 46 & 87 & 82 & 18 & 21 & 1,600 & & 300 & \\
\hline-0.76 & -1.34 & & & +4 & +13 & -18 & -21 & +3 & +4 & -800 & & 0 & \\
\hline 4.36 & 9.03 & 5 & 9 & 25 & 44 & 91 & 130 & 12 & 14 & 1,300 & 1,600 & 200 & 400 \\
\hline 4.01 & 5.81 & 11 & 11 & 44 & 48 & 88 & 89 & 30 & 30 & 1,100 & 800 & 200 & 200 \\
\hline 7.06 & 7.40 & 5 & & 29 & 28 & 95 & 88 & $21 t$ & $25 \ddagger$ & 2,000 & 1,200 & 200 & 100 \\
\hline 6.22 & 7.32 & & 15 & 38 & 52 & 90 & 100 & $23 t$ & $31 t$ & 1,600 & 1,400 & 300 & 300 \\
\hline-0.84 & -0.08 & & & +9 & +24 & -5 & +12 & +2 & +6 & -400 & +200 & +100 & +200 \\
\hline 6.60 & 10.98 & 4 & & 25 & 35 & 78 & 86 & 14 & 25 & 2,400 & 2,300 & 300 & 300 \\
\hline 6.15 & 9.74 & & & & & 104 & 108 & $12 \ddagger$ & $16 \ddagger$ & 2,700 & 2,900 & & \\
\hline 5.93 & 7.76 & & & & & 88 & 95 & $16 \ddagger$ & $17 \ddagger$ & 2,200 & 2,300 & & \\
\hline-0.22 & -1.98 & & & & & -16 & -13 & +4 & +1 & -500 & -600 & & \\
\hline 6.35 & 7.06 & 5 & & 46 & 51 & 132 & 134 & & & 2,400 & 2,200 & & \\
\hline 6.13 & 6.50 & 4 & 5 & 39 & 49 & 130 & 134 & & & 2,500 & 2,400 & & \\
\hline-0.22 & -0.56 & -1 & & -7 & -2 & -2 & 0 & & & +100 & +200 & & \\
\hline 6.00 & & & & 38 & & 138 & & $11 \ddagger$ & & 2.800 & & & \\
\hline 6.33 & & & & 39 & & 145 & & $11 t$ & & 2,500 & & & \\
\hline 6.76 & & & & 53 & & 144 & & $27 \ddagger$ & & 2,500 & & & \\
\hline+0.43 & & & & +14 & & -1 & & +16 & & 0 & & & \\
\hline
\end{tabular}


TABLE II-Continued

\begin{tabular}{|c|c|c|c|c|c|c|c|c|c|c|c|c|}
\hline \multirow{2}{*}{\multicolumn{2}{|c|}{ Patient }} & \multirow{3}{*}{$\frac{\text { BSA }}{m^{2}}$} & & & \multicolumn{2}{|c|}{$\dot{V}_{0}$} & \multicolumn{2}{|c|}{$\dot{\mathrm{Q}} \mathrm{i}$} & \multicolumn{2}{|c|}{ Ve } & \multicolumn{2}{|c|}{ Heart rate } \\
\hline & & & \multicolumn{2}{|c|}{ Rhythm } & $\mathbf{R}$ & $\mathrm{E}$ & $\mathbf{R}$ & $\mathrm{E}$ & $\mathbf{R}$ & $\mathrm{E}$ & $\mathbf{R}$ & $\mathrm{E}$ \\
\hline & & & & & \multicolumn{2}{|c|}{$\mathrm{ml} / \mathrm{min} / \mathrm{m}^{2}$} & \multicolumn{2}{|c|}{$L / \min / m^{2}$} & \multicolumn{2}{|c|}{$\mathrm{ml} / \mathrm{beat} / \mathrm{m}^{2}$} & \multicolumn{2}{|c|}{ beats/min } \\
\hline \multirow[t]{3}{*}{23.} & A.E. & 1.68 & & $\mathrm{AF}$ & 117 & 165 & 2.89 & 3.22 & 30 & 33 & 96 & 99 \\
\hline & & & $+1 \mathrm{hr}$ & NSR & 118 & 197 & 2.71 & 3.24 & 38 & 42 & 72 & 78 \\
\hline & & & & $\Delta$ & +1 & +32 & -0.18 & +0.02 & +8 & +9 & -24 & -21 \\
\hline \multirow[t]{3}{*}{25.} & W.F. & 2.10 & & $\mathrm{AF}$ & 127 & 446 & 1.91 & 4.03 & 19 & 22 & 103 & 180 \\
\hline & & & $+1 \mathrm{hr}$ & NSR & 133 & 383 & 2.84 & 4.93 & 36 & 49 & 80 & 100 \\
\hline & & & & $\Delta$ & +6 & -63 & +0.93 & +0.90 & +17 & +27 & -23 & -80 \\
\hline \multirow[t]{9}{*}{26} & H.B. & 2.06 & & $\mathrm{AF}$ & 133 & 237 & 2.46 & 3.03 & 18 & 20 & 135 & 151 \\
\hline & & & $+1 \mathrm{hr}$ & NSR & 128 & 242 & 2.62 & 3.68 & 28 & 36 & 93 & 100 \\
\hline & & & & $\Delta$ & -5 & +5 & +0.16 & +0.65 & +10 & +16 & -42 & -51 \\
\hline & & Mean & & $\mathrm{AF}$ & 137 & 230 & 2.30 & 2.98 & 23 & 25 & 111 & 127 \\
\hline & & $\mathrm{SE}$ & & & \pm 4 & \pm 21 & \pm 0.14 & \pm 0.16 & \pm 2 & \pm 2 & \pm 9 & \pm 8 \\
\hline & & Mean & & NSR & 136 & 217 & 2.54 & 3.20 & 30 & 33 & 87 & 98 \\
\hline & & $\mathrm{SE}$ & & & \pm 5 & \pm 18 & \pm 0.10 & \pm 0.21 & \pm 2 & \pm 2 & \pm 4 & \pm 4 \\
\hline & & Mean & & $\Delta$ & -1 & -14 & $+0.24 \S$ & $+0.22 \|$ & $+7 \pi$ & $+8 \pi$ & $-24 \pi$ & $-28 q$ \\
\hline & & $\mathrm{SE}$ & & & \pm 3 & \pm 8 & \pm 0.09 & \pm 0.09 & \pm 2 & \pm 2 & \pm 7 & \pm 6 \\
\hline \multicolumn{13}{|c|}{ B. Patients not converted } \\
\hline \multirow[t]{3}{*}{16.} & M.I. & 1.37 & & $\mathrm{AF}$ & 114 & 274 & 1.93 & 3.26 & 32 & 34 & 60 & 96 \\
\hline & & & $+1 \mathrm{hr}$ & $\mathrm{AF}$ & 104 & 259 & 1.86 & 3.13 & 41 & 35 & 45 & 90 \\
\hline & & & & $\Delta$ & -10 & -15 & -.07 & -.13 & 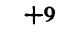 & +1 & -15 & -6 \\
\hline \multirow[t]{4}{*}{17.} & B.D. & 1.51 & $-48 \mathrm{mos}$ & $\mathrm{AF} \dagger$ & 117 & 296 & 2.15 & 3.34 & 30 & 27 & 71 & 123 \\
\hline & & 1.42 & & $\mathrm{AF}$ & 139 & 300 & 2.54 & 3.36 & 36 & 31 & 70 & 110 \\
\hline & & & $+1 \mathrm{hr}$ & $\mathrm{AF}$ & 140 & 270 & 2.88 & 4.00 & 41 & 40 & 70 & 100 \\
\hline & & & & $\Delta$ & +1 & -30 & +0.34 & +0.64 & +5 & +9 & 0 & -10 \\
\hline \multirow[t]{4}{*}{18.} & M.H. & 1.52 & & $\mathrm{AF}$ & 136 & 222 & 2.19 & 2.73 & 28 & 24 & 78 & 111 \\
\hline & & & $+1 \mathrm{hr}$ & $\mathrm{AF}$ & 102 & 224 & 1.86 & 2.73 & 22 & 28 & 84 & 96 \\
\hline & & & +6 days & AF & & & 1.96 & 2.25 & 22 & 27 & 90 & 84 \\
\hline & & & & $\Delta$ & -34 & +2 & -0.33 & 0.00 & -6 & +4 & +6 & -15 \\
\hline \multirow[t]{9}{*}{24.} & c.s. & 1.72 & & $\mathrm{AF}$ & 156 & 278 & 3.22 & 3.82 & 33 & 34 & 96 & 110 \\
\hline & & & $+1 \mathrm{hr}$ & $\mathrm{AF}$ & 156 & 255 & 3.48 & 4.19 & 35 & 42 & 100 & 100 \\
\hline & & & & $\Delta$ & 0 & -23 & +0.26 & +0.37 & +2 & +8 & +4 & -10 \\
\hline & & Mean & & $\mathrm{AF}$ & 136 & 269 & 2.47 & & 32 & 31 & 76 & 107 \\
\hline & & $\mathrm{SE}$ & & & \pm 9 & \pm 17 & \pm 0.28 & \pm 0.22 & \pm 2 & \pm 2 & \pm 8 & \pm 4 \\
\hline & & Mean & $+1 \mathrm{hr}$ & $\mathrm{AF}$ & 126 & 252 & 2.52 & 3.51 & 35 & 36 & 75 & 97 \\
\hline & & $\mathrm{SE}$ & & & \pm 13 & \pm 10 & \pm 0.40 & \pm 0.35 & \pm 4 & \pm 3 & \pm 12 & \pm 2 \\
\hline & & Mean & & $\Delta$ & -11 & -17 & +.05 & +0.22 & +3 & +6 & -1 & $-10 \S$ \\
\hline & & $\mathrm{SE}$ & & & \pm 8 & \pm 7 & \pm 0.27 & \pm 0.17 & \pm 3 & \pm 2 & \pm 5 & \pm 2 \\
\hline
\end{tabular}

* Abbreviations $: \dot{V}_{O}=$ oxygen consumption; $\dot{Q i}=$ cardiac output per square meter of body surface area; VE $=$ stroke volume; a-v = arteriovenous; $\overline{P A}=$ mean right atrial pressure; $P P_{A A}=$ mean pulmonary arterial pressure; $\mathbf{P} \overline{\mathrm{BA}}=$ mean peripheral arterial pressure; $P$ wedge $=$ mean wedge (or left atrialt) pressure; $R \mathbf{R}=$ total systemic vascular resistance; $R$ Part $=$ pulmonary arteriolar resistance. $R=$ at rest; $E=$ at exercise. $\mathrm{AF}=$ atrial fibrillation; $\mathrm{A} . \mathrm{Fl}$. = atrial flutter; NSR = normal sinus rhythm. $\Delta=$ difference in determination immediately before and 1 hour

vanometers with a $1-\mathrm{m}$ light path. At a recording sensitivity of $1 \mathrm{~mm} \mathrm{Hg}=1 \mathrm{~mm}$ deflection, the catheter-tipto-paper frequency response of this system is flat to 19 cps when tested with a hydraulic pressure generator.

Data obtained in other patients with heart disease studied at rest, during exercise, and one-half hour after exercise were used for comparison of the rest-"rerest" studies in the present series. Seven of these patients had ultimately fatal myocardial disease of various etiologies (11), and seven had chronic rheumatic mitral disease.

\section{Results}

The clinical consequences of the attempts at conversion are summarized in Table I. In Table
II the hemodynamic data obtained in these patients are presented. In addition to the results of the studies immediately before and 1 hour after awakening from the conversion attempt and their differences, indicated by the $\Delta$ values, data from any earlier or later studies are included for comparison; intervening valvular surgical intervention is indicated. Data from the four patients who did not convert are shown separately. Whenever oxygen consumption data are not shown, the cardiac outputs were obtained by the indicatordilution technique. The means and standard er- 
TABLE II-Continued

\begin{tabular}{|c|c|c|c|c|c|c|c|c|c|c|c|c|c|}
\hline \multicolumn{2}{|c|}{ a-v difference } & \multicolumn{2}{|c|}{$\mathrm{P} \overline{\mathrm{RA}}$} & \multicolumn{2}{|c|}{$\overline{P A}$} & \multicolumn{2}{|c|}{$\mathrm{P} \overline{\mathrm{BA}}$} & \multicolumn{2}{|c|}{ Pwedge } & \multicolumn{2}{|c|}{ Rs } & \multicolumn{2}{|c|}{ Rpart. } \\
\hline $\mathbf{R}$ & $\mathrm{E}$ & $\mathbf{R}$ & $\mathrm{E}$ & $\mathbf{R}$ & $\mathrm{E}$ & $\mathbf{R}$ & $\mathrm{E}$ & $\mathbf{R}$ & $\mathrm{E}$ & $\mathbf{R}$ & $\mathrm{E}$ & $\mathbf{R}$ & $\mathrm{E}$ \\
\hline \multicolumn{2}{|c|}{$\mathrm{ml} / 100 \mathrm{ml}$} & \multicolumn{2}{|c|}{$m m \mathrm{Hg}$} & \multicolumn{2}{|c|}{$m m ~ H g$} & \multicolumn{2}{|c|}{$m m ~ H g$} & \multicolumn{2}{|c|}{$m m \mathrm{Hg}$} & \multicolumn{2}{|c|}{ dyne-sec-cm ${ }^{-5}$} & \multicolumn{2}{|c|}{ dyne-sec- $\mathrm{cm}^{-\mathrm{s}}$} \\
\hline 4.03 & 5.12 & 7 & & 27 & 38 & 88 & 100 & 18 & & 1,400 & 1,500 & 100 & \\
\hline 4.37 & 6.08 & & 9 & 31 & 34 & 93 & 93 & & & 1,600 & 1,400 & & \\
\hline+0.34 & +0.96 & & & +4 & -4 & +5 & -7 & & & +200 & -100 & & \\
\hline 6.65 & 11.05 & 7 & & 10 & 24 & 95 & 111 & $9+$ & $20+$ & 1,900 & 1,000 & 100 & 100 \\
\hline 4.70 & 7.77 & & & & & 94 & 102 & $2+$ & $4+$ & 1,300 & 800 & & \\
\hline-1.95 & -3.28 & & & & & -1 & -9 & -7 & -16 & -600 & -200 & & \\
\hline 5.44 & 7.84 & 7 & & 30 & 36 & 141 & 143 & $13 \ddagger$ & $13+$ & 2,200 & 1,800 & 200 & 200 \\
\hline 4.89 & 6.60 & & & 24 & 36 & 125 & 136 & $7 \ddagger$ & $11 \ddagger$ & 1,900 & 1,400 & 100 & 100 \\
\hline-0.55 & -1.24 & & & -6 & 0 & -16 & -7 & -6 & -2 & -300 & -400 & -100 & -100 \\
\hline 5.96 & 7.88 & 8 & 9 & 30 & 37 & 99 & 99 & 14 & 18 & 2,012 & 1,586 & 220 & 150 \\
\hline \pm 0.27 & \pm .47 & \pm 1 & \pm 1 & \pm 2 & \pm 3 & \pm 5 & \pm 5 & \pm 1 & \pm 2 & \pm 111 & \pm 134 & \pm 37 & \pm 50 \\
\hline 5.47 & 6.92 & 5 & 8 & 32 & 40 & 95 & 99 & 15 & 17 & 1,759 & 1,529 & 220 & 200 \\
\hline \pm 0.22 & \pm 0.21 & \pm 1 & \pm 1 & \pm 3 & \pm 3 & \pm 5 & \pm 4 & \pm 3 & \pm 5 & \pm 106 & \pm 119 & \pm 49 & \pm 100 \\
\hline$-.50 \S$ & $-0.96 \pi$ & -2 & -1 & +2 & +4 & -4 & 0 & +1 & -1 & $-253 \S$ & -57 & 0 & +50 \\
\hline $\pm 0.18^{\circ}$ & \pm 0.30 & \pm 1 & 0 & \pm 2 & \pm 3 & \pm 2 & \pm 3 & \pm 3 & \pm 4 & \pm 91 & \pm 66 & \pm 32 & \pm 150 \\
\hline 5.89 & 8.43 & 3 & & 16 & 24 & 85 & 99 & $10_{+}^{+}$ & $17+$ & 2,600 & 1,800 & 200 & 100 \\
\hline 5.58 & 8.28 & & 6 & 17 & 24 & 78 & 81 & $15_{+}^{+}$ & $18_{+}^{+}$ & 2,500 & 1,500 & 100 & 100 \\
\hline-.31 & -.15 & & & +1 & 0 & -7 & -18 & +5 & +1 & -100 & -300 & -100 & 0 \\
\hline 5.46 & 8.83 & 8 & 11 & 30 & 55 & 91 & 99 & $22 \ddagger$ & & 2,300 & 1,600 & 200 & \\
\hline 5.46 & 8.89 & $y$ & & 42 & 58 & 96 & 103 & $22+$ & $45_{+}^{+}$ & 2,100 & 1,800 & 400 & 200 \\
\hline 4.86 & 6.76 & & 11 & 39 & 69 & 107 & 110 & $29+$ & $39+$ & 2,100 & 1,600 & 200 & 400 \\
\hline-0.60 & -2.13 & & & -3 & +11 & +11 & +7 & +7 & -6 & 0 & -200 & -200 & +200 \\
\hline 6.20 & 8.14 & 9 & & 30 & 52 & 100 & 112 & 19 & & 2,400 & 2,200 & 300 & \\
\hline 5.52 & 8.21 & & & 33 & 43 & 86 & 96 & $10^{+}$ & $20^{+}$ & 2,400 & 1,800 & & \\
\hline-0.68 & +0.07 & & & +3 & -9 & -14 & -16 & $19+$ & $20_{+}$ & 0 & -400 & & \\
\hline 4.85 & 7.32 & & & 23 & 31 & 92 & 89 & & & 1,300 & 1,100 & & \\
\hline 4.49 & 6.09 & & & 28 & 38 & 78 & 88 & & & 1,000 & 1,000 & & \\
\hline-0.36 & -1.23 & & & +5 & +7 & -14 & -1 & & & -300 & -100 & & \\
\hline 5.60 & 8.20 & & & 28 & 41 & 93 & 101 & 16 & 31 & 2,100 & 1,725 & 300 & 150 \\
\hline \pm 0.29 & \pm 0.33 & & & \pm 6 & \pm 8 & \pm 3 & \pm 5 & \pm 6 & \pm 14 & \pm 286 & \pm 229 & \pm 100 & \pm 50 \\
\hline 5.11 & 7.34 & & & 29 & 44 & 87 & 94 & 22 & 29 & 2,000 & 1,475 & 150 & 250 \\
\hline \pm 0.27 & \pm 0.54 & & & \pm 5 & \pm 9 & \pm 7 & \pm 6 & \pm 7 & \pm 11 & \pm 344 & \pm 171 & \pm 50 & \pm 150 \\
\hline$-0.49 \S$ & -0.86 & & & +2 & +2 & -6 & -7 & +6 & -3 & -100 & $-250 \|$ & -150 & +100 \\
\hline \pm 0.09 & \pm 0.51 & & & \pm 2 & \pm 4 & \pm 6 & \pm 6 & \pm 1 & \pm 4 & \pm 71 & \pm 65 & \pm 50 & \pm 100 \\
\hline
\end{tabular}

rors of the means before and after conversion are shown.

Cardiac output, oxygen consumption, and arteriovenous oxygen difference. Slight but significant increases in mean cardiac output of $0.24 \mathrm{~L}$ per minute per $\mathrm{m}^{2} \pm \mathrm{SD} 0.38$ at rest and $0.22 \mathrm{~L}$ per minute per $\mathrm{m}^{2} \pm \mathrm{SD} 0.33$ at exercise were found 1 hour after awakening for the group of sixteen patients who converted. No change in oxygen consumption was found, but a significant narrowing of systemic arteriovenous oxygen difference occurred. In the four patients who did not convert, however, although no change in either cardiac index or oxygen consumption occurred, a significant narrowing of arteriovenous oxygen difference was also noted. Since the experimental protocol to which all of these patients were subjected was quite long and included light anesthesia and the transthoracic shock (s), it was felt that changes during the protocol, unrelated to any change in rhythm, might have occurred. Therefore, restrerest data obtained in 14 other patients with chronic heart disease during output measurements $\frac{1}{2}$ hour after intervening exercise (Figure 1a) were plotted as a basis for comparison with the rest-postconversion attempt and the rest-pre- 


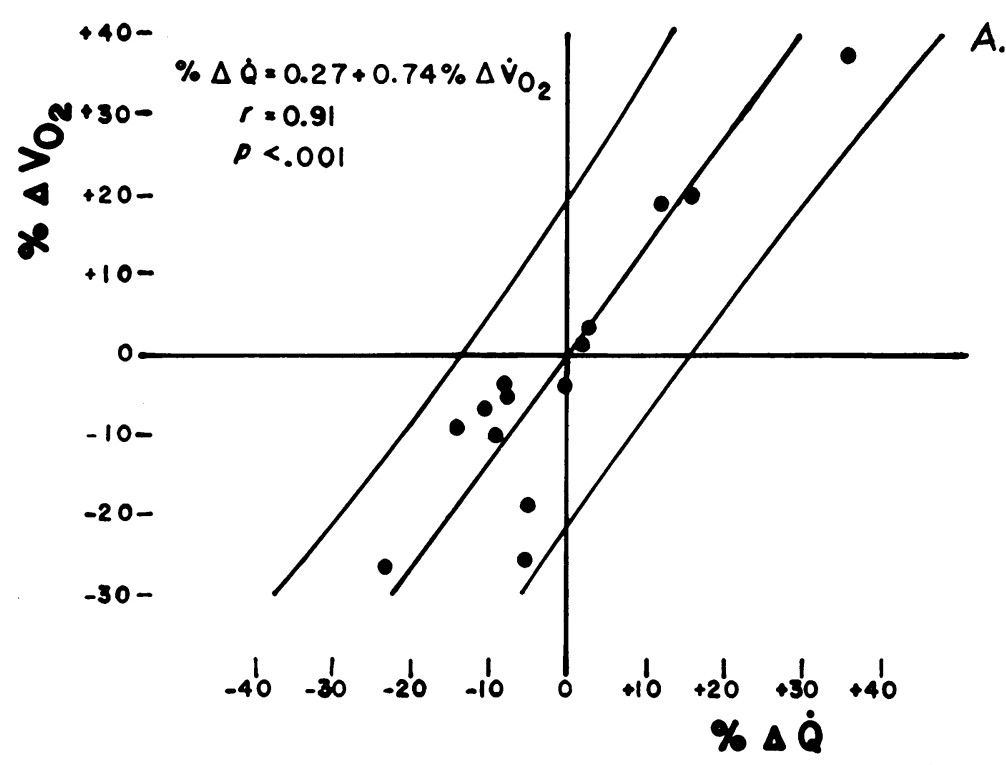

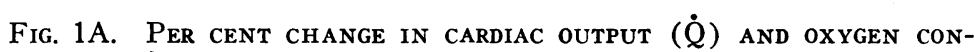
SUMPTION $\left(\dot{\mathrm{V}} \mathrm{O}_{2}\right)$ IN REREST STUDIES PERFORMED ONE-HALF HOUR AFTER EXERCISE OUTPUT STUDIES IN 14 PATIENTS WITH heART DISEASE. The regression line and lines defining $2 \mathrm{SD}$ of the relationship are shown.

B.

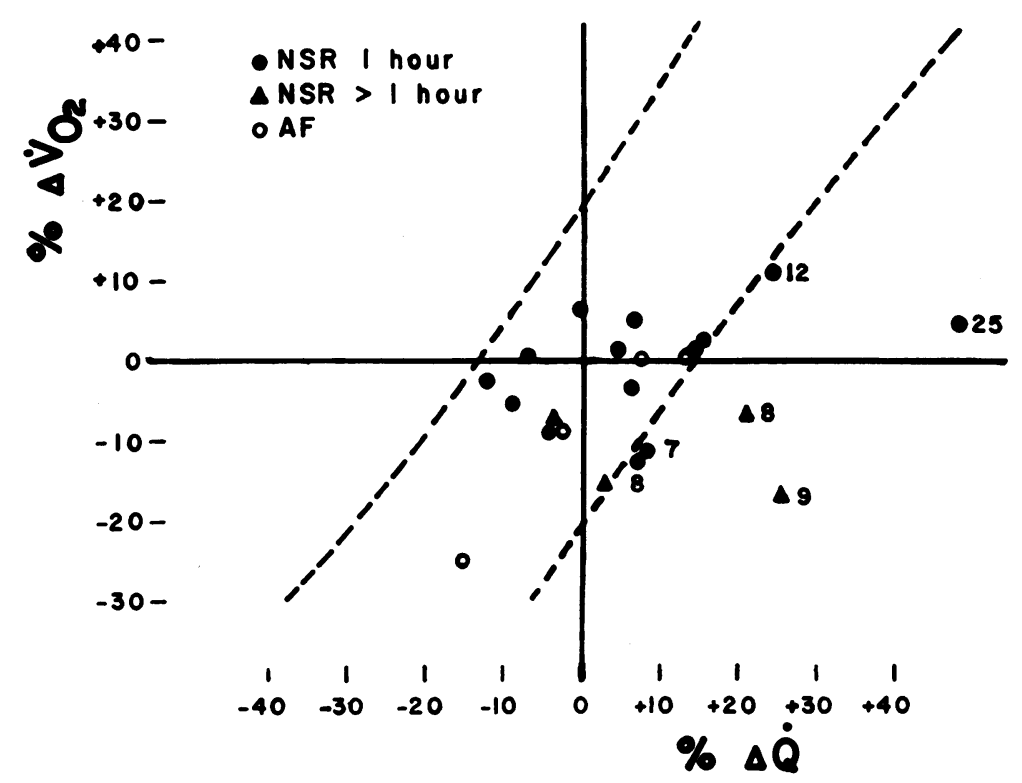

Fig. 1B. Per Cent change in $\dot{Q}$ and $\dot{V} \mathrm{O}_{2}$ IN THE Rerest studies in 14 PATIENTS AFTER CONVERSION TO NORMAL SINUS RHYTHM AND IN FOUR PATIENTS WHO DID NOT CONVERT. The dotted lines define 2 SD of the relationship shown in Figure 1A. NSR = normal sinus rhythm; $A F=$ atrial fibrillation. 
conversion attempt output data of the present series (Figure 1b). The $95 \%$ confidence limits of the regression plotted in Figure la are shown as dashed lines in Figure 1b. It will be noted in Figure 1b that at 1 hour after the conversion attempt the output changes of ten of 14 patients who converted and of the four who did not convert fell within the range of change that was found in the 14 patients not subjected to the present protocol. Only four patients (No. 7, 8, 12, and 25) had output changes greater with respect to oxygen consumption than did the four patients who did not convert and the 14 not participating in the conversion attempt.

The per cent changes in cardiac output with respect to changes in oxygen consumption during exercise after the conversion attempt compared with those before conversion are plotted in Figure 2. We do not have re-exercise-exercise data from other patients for comparison and can only compare the data from the patients who converted with those of the four who did not. Only one patient (No. 25) had a greater change of cardiac output with respect to change in oxygen consumption after conversion than did the patients who did not convert.

Heart rate and stroke volume. A significant decrease in mean heart rate and an increase in mean stroke volume were found both at rest and during exercise in the patients who converted. In the four who did not convert, the heart rate at exercise after the conversion attempt was also significantly lower than before the attempt. Since a stroke volume increase would be expected with a heart rate decrease even in the absence of an increase in cardiac output, the patients who converted were divided into two groups for analysis of the relationship of heart rate to cardiac output change, as shown in Table III. In the table the data in patients in whom a heart rate decrease of more than 30 beats per minute had occurred at rest or during exercise after conversion (A) are compared with those in whom a rate change of lesser magnitude was noted (B). In group A a significant decrease in heart rate and increase in stroke volume occurred both at rest and during exercise but only during exercise in group $B$. In group A a significant increase in cardiac output

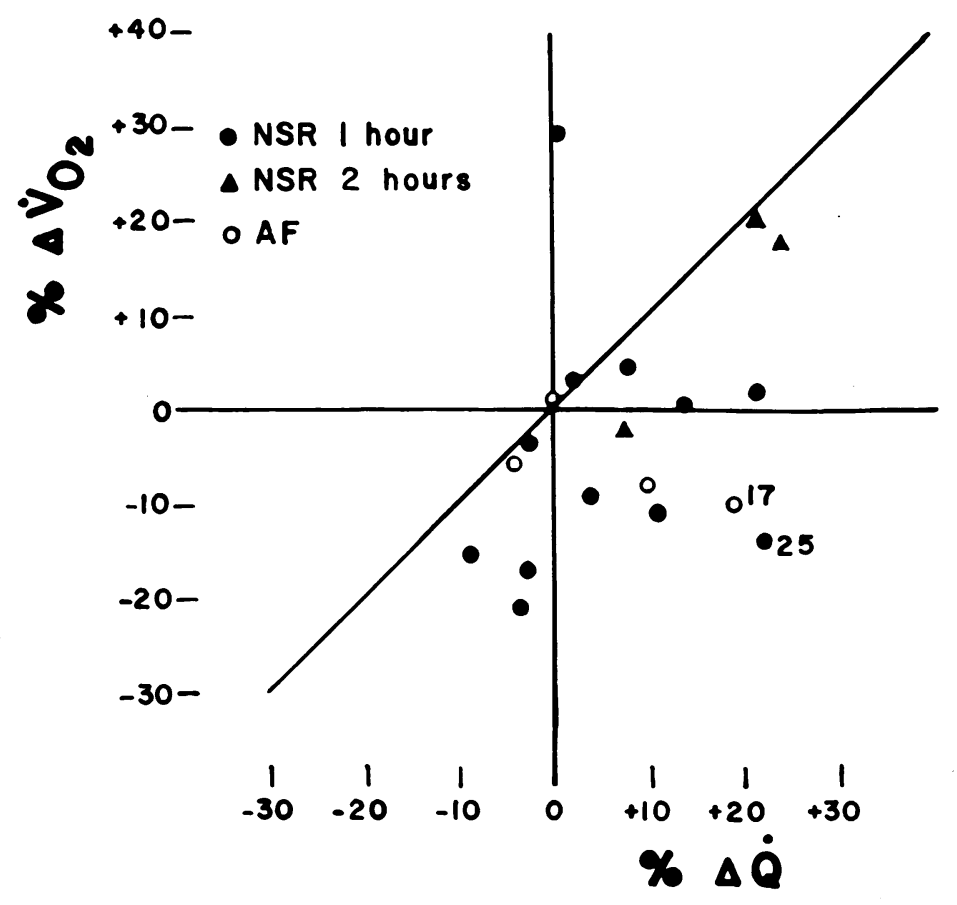

Fig. 2. Per cent change in $Q$ and $\mathrm{VO}_{2}$ DURing RE-exercise After CONVERSION TO NORMAL SINUS RHYTHM IN 13 PATIENTS COMPARED WITH THE FOUR PATIENTS WHO DID NOT CONVERT. 
TABLE III

Effect on hemodynamic data of change in heart rate after conversion

\begin{tabular}{|c|c|c|c|c|c|c|}
\hline & \multicolumn{3}{|c|}{$\begin{array}{c}\text { Rest } \\
(3,5,7,11,12,26) * \\
\end{array}$} & \multicolumn{3}{|c|}{$\begin{array}{c}\text { Exercise } \\
(5,7,11,25,26)^{*} \\
\end{array}$} \\
\hline & $\mathrm{AF}$ & NSR & $\Delta$ & $\mathrm{AF}$ & NSR & $\Delta$ \\
\hline \multicolumn{7}{|c|}{ A. Decrease in heart rate more than 30 beats/minute } \\
\hline $\begin{array}{l}\text { Oxygen consumption, } \\
\mathrm{ml} / \mathrm{min} / \mathrm{m}^{2}\end{array}$ & $143 \pm 5$ & $143 \pm 7$ & 0 & $278 \pm 57$ & $259 \pm 42$ & $-19 \pm 3 \dagger$ \\
\hline $\begin{array}{l}\text { Cardiac output, } \\
\mathrm{L} / \mathrm{min} / \mathrm{m}^{2}\end{array}$ & $2.13 \pm 0.14$ & $2.59 \pm 0.07$ & $+0.46 \pm 0.14 \ddagger$ & $3.10 \pm 0.24$ & $3.65 \pm 0.36$ & $+0.55 \pm 0.17 \ddagger$ \\
\hline $\begin{array}{l}\text { Stroke volume, } \\
\quad \mathrm{ml} / \text { beat } / \mathrm{m}^{2}\end{array}$ & $15 \pm 2$ & $29 \pm 4$ & $+14 \pm 2 \dagger$ & $19 \pm 1$ & $34 \pm 5$ & $+15 \pm 4 \S$ \\
\hline $\begin{array}{l}\text { Heart rate, } \\
\text { beats/min }\end{array}$ & $152 \pm 16$ & $96 \pm 10$ & $-56 \pm 10 \dagger$ & $157 \pm 11$ & $104 \pm 7$ & $-53 \pm 7 \dagger$ \\
\hline $\begin{array}{l}\text { a-v difference } \\
\mathrm{ml} / 100 \mathrm{ml}\end{array}$ & $6.24 \pm 0.32$ & $5.57 \pm 0.28$ & $-0.67 \pm 0.23$ & $8.64 \pm 0.62$ & $7.09 \pm 0.20$ & $-1.55 \pm 0.44 \ddagger$ \\
\hline
\end{tabular}

B. Decrease in heart rate less than 30 beats/minute

\begin{tabular}{|c|c|c|c|c|c|c|}
\hline \multirow[b]{2}{*}{$\begin{array}{l}\text { Oxygen consumption, } \\
\mathrm{ml} / \mathrm{min} / \mathrm{m}^{2}\end{array}$} & \multicolumn{3}{|c|}{$(6,8,9 a, b, 10,13,14,21,22,23,25)^{*}$} & \multicolumn{3}{|c|}{$(6,8,9 a, b, 10,13,14,21,23)^{*}$} \\
\hline & $135 \pm 5$ & $133 \pm 6$ & $-2 \pm 1$ & $207 \pm 10$ & $196 \pm 12$ & $-11 \pm 9$ \\
\hline $\begin{array}{l}\text { Cardiac output, } \\
\mathrm{L} / \mathrm{min} / \mathrm{m}^{2}\end{array}$ & $2.39 \pm 0.20$ & $2.51 \pm 0.16$ & $+0.12 \pm 0.11$ & $2.92 \pm 0.22$ & $2.95 \pm 0.24$ & $+0.03 \pm 0.06$ \\
\hline $\begin{array}{l}\text { Stroke volume, } \\
\mathrm{ml} / \text { beat } / \mathrm{m}^{2}\end{array}$ & $27 \pm 2$ & $31 \pm 2$ & $+4 \pm 2$ & $28 \pm 3$ & $32 \pm 3$ & $+4 \pm 1 \ddagger$ \\
\hline $\begin{array}{l}\text { Heart rate, } \\
\text { beats } / \text { min }\end{array}$ & $89 \pm 3$ & $82 \pm 3$ & $-7 \pm 4$ & $106 \pm 5$ & $94 \pm 4$ & $-12 \pm 4 \S$ \\
\hline $\begin{array}{l}\text { a-v difference, } \\
\mathrm{ml} / 100 \mathrm{ml}\end{array}$ & $5.86 \pm 0.35$ & $5.43 \pm 0.28$ & $-0.43 \pm 0.24$ & $7.40 \pm 0.62$ & $6.81 \pm 0.32$ & $-0.59 \pm 0.35$ \\
\hline
\end{tabular}

* Patient-study number.

$\dagger p<0.01$.

$\ddagger p<0.05$.

$\S p<0.02$.

was also observed, i.e., the stroke volume increase was greater than that which might have been anticipated on the basis of the rate decrease. In group B at rest, however, no significant change in heart rate, stroke volume, or output was found, and during exercise the stroke volume increased only reciprocally to the slight rate decrease, i.e., no output increase occurred.

The degree of irregularity of ventricular rhythm, expressed as the standard error of the RR intervals in the electrocardiograms recorded during each Fick output, was not related to the observed changes in stroke volume or cardiac output. Furthermore, there was no constant relationship between the change in output and the absolute level of output before conversion.

The output and rate changes observed immediately before and after conversion during the light thiopental anesthesia (not shown in the tables) were not significantly different from those while awake.
In studies made at longer intervals after conversion, one patient (No. 5) with mitral stenosis had no further increase in output or stroke volume at 1 or 11 months, despite a markedly lower rate; another (No. 8), however, had a major increase in output at 2 hours and a further increase to normal at 3 months, attributable to a stroke volume increase without any rate change. One other patient (No. 9) had an increase of $0.60 \mathrm{~L}$ per minute per $\mathrm{m}^{2}$ by 2 hours without a rate change.

Atrial pressure. Mean right atrial pressure decreased in each of the four patients in whom it was measured before and after conversion at rest and also in the three in whom it was measured during exercise. The "a" waves that developed were quite small; a sharper " $c$ " wave and $\mathrm{x}$-descent were regularly seen.

Mean left atrial or pulmonary arterial wedge pressure was slightly higher after conversion in four of five patients with rheumatic heart disease in whom changes were available at rest and in 
three of four at exercise; left atrial pressure was also much higher at rest in one patient with hypertensive heart disease. By contrast, in the two patients with atrial fibrillation as the only sign of possible heart disease definite decreases occurred after conversion. As was seen in the right atrial pressure contours, surprisingly small "a" waves appeared after restoration of sinus rhythm, but the $\mathrm{x}$-descent became clearer (Figure 3 ).

Pulmonary arterial pressure and arteriolar resistance. Mean pulmonary arterial pressure was elevated in the majority of the patients and did not clearly change with conversion. Pulmonary arteriolar resistances similarly showed no consistent change.

Brachial arterial pressure and total systemic resistance. Mean brachial arterial pressure changed little; systemic resistance, therefore, tended to decrease only in those patients with major output increases.

Left ventricular function. In Table IV measurements made in the left ventricles of five of these patients are presented. In three, two with mitral stenosis (No. 13 and 14) and one with hypertensive heart disease (No. 22), increases in both ventricular mean-diastolic and end-diastolic

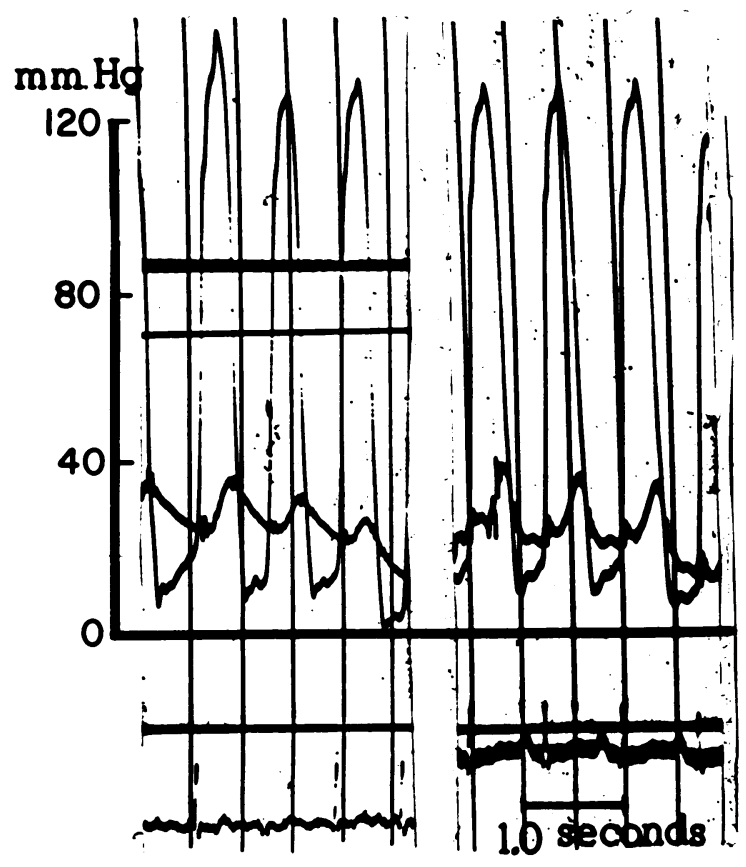

Fig. 3. Simultaneovs left atrial (transeptal) AND Left ventricllar (Retrograde) PREsSlres aNd the ELECTROCARdIOGRAM iN a PATIENT With Mitral Stexosis With ATRIAL Fibrillation (LefT) AND $1 \frac{1}{2}$ HOLRS AFter CONVERSION TO SINCS RHYTHM (RIGHT).

TABLE IV

Left ventricular function changes accompanying conversion from atrial fibrillation to normal sinus rhythm*

\begin{tabular}{|c|c|c|c|c|c|c|c|c|c|c|c|c|c|}
\hline \multirow[b]{2}{*}{ Patient } & \multirow[b]{2}{*}{ Rhythm } & \multicolumn{2}{|c|}{$\mathrm{VE}$} & \multicolumn{2}{|c|}{ Heart rate } & \multicolumn{2}{|c|}{ PLVD } & \multicolumn{2}{|c|}{ Plved } & \multicolumn{2}{|c|}{ Plved-Plid } & \multicolumn{2}{|c|}{$\dot{\mathbf{W}}_{\mathrm{LV}}$} \\
\hline & & $\mathrm{R}$ & $\mathrm{E}$ & $\mathbf{R}$ & $\mathrm{E}$ & $\mathrm{R}$ & $\mathrm{E}$ & $\mathbf{R}$ & $\mathrm{E}$ & $\mathrm{R}$ & $\mathrm{E}$ & $\mathrm{R}$ & $\mathrm{E}$ \\
\hline & & \multicolumn{2}{|c|}{$m l / b e a t$} & \multicolumn{2}{|c|}{ beats/min } & \multicolumn{2}{|c|}{$m m ~ H g$} & \multicolumn{2}{|c|}{$m m \mathrm{Hg}$} & \multicolumn{2}{|c|}{$m m \mathrm{Hg}$} & \multicolumn{2}{|c|}{ ergs $\times 10^{6}$ beal } \\
\hline 13. C.B. & $\begin{array}{l}\mathrm{AF} \\
\underset{\Delta}{\mathrm{NSR}}\end{array}$ & $\begin{array}{r}43 \\
56 \\
+13\end{array}$ & $\begin{array}{r}56 \\
63 \\
+7\end{array}$ & $\begin{array}{r}90 \\
78 \\
-12\end{array}$ & $\begin{array}{r}102 \\
90 \\
-12\end{array}$ & $\begin{array}{r}10 \\
14 \\
+4\end{array}$ & $\begin{array}{r}13 \\
13 \\
0\end{array}$ & $\begin{array}{r}12 \\
16 \\
+4\end{array}$ & $\begin{array}{r}16 \\
16 \\
0\end{array}$ & $\begin{array}{r}+2 \\
+2 \\
0\end{array}$ & $\begin{array}{r}+3 \\
+3 \\
0\end{array}$ & $\begin{array}{r}4.4 \\
5.0 \\
+0.6\end{array}$ & $\begin{array}{r}7.3 \\
8.7 \\
+1.4\end{array}$ \\
\hline 14. L.A. & $\begin{array}{l}\mathrm{AF} \\
\underset{\Delta}{N S R}\end{array}$ & $\begin{array}{r}38 \\
40 \\
+2\end{array}$ & $\begin{array}{r}30 \\
37 \\
+7\end{array}$ & $\begin{array}{r}81 \\
81 \\
0\end{array}$ & $\begin{array}{r}99 \\
90 \\
-9\end{array}$ & $\begin{array}{r}3 \\
5 \\
+2\end{array}$ & $\begin{array}{r}3 \\
4 \\
+1\end{array}$ & $\begin{array}{r}5 \\
9 \\
+4\end{array}$ & $\begin{array}{r}6 \\
7 \\
+1\end{array}$ & $\begin{array}{l}+2 \\
+4 \\
+2\end{array}$ & $\begin{array}{r}+3 \\
+3 \\
0\end{array}$ & $\begin{array}{r}4.9 \\
4.6 \\
-0.3\end{array}$ & $\begin{array}{r}4.7 \\
5.4 \\
+0.7\end{array}$ \\
\hline 22. A.A. & $\begin{array}{l}A F \\
\text { AF } \\
\text { NSR } \\
\Delta\end{array}$ & $\begin{array}{r}43 \\
51 \\
57 \\
+6\end{array}$ & & $\begin{array}{r}90 \\
90 \\
80 \\
-10\end{array}$ & & $\begin{array}{r}10 \\
25 \\
+15\end{array}$ & & $\begin{array}{r}11 \\
11 \\
27 \\
+16\end{array}$ & & $\begin{array}{l}+1 \\
+2 \\
+1\end{array}$ & & $\begin{array}{r}9.7 \\
10.3 \\
12.8 \\
+2.5\end{array}$ & \\
\hline 25. W.F. & $\begin{array}{l}\mathrm{AF} \\
\underset{\Delta}{\mathrm{ASR}}\end{array}$ & $\begin{array}{r}40 \\
75 \\
+35\end{array}$ & $\begin{array}{r}47 \\
103 \\
+56\end{array}$ & $\begin{array}{r}103 \\
80 \\
-23\end{array}$ & $\begin{array}{r}180 \\
100 \\
-80\end{array}$ & $\begin{array}{r}8 \\
2 \\
-6\end{array}$ & $\begin{array}{r}8 \\
4 \\
-4\end{array}$ & $\begin{array}{r}8 \\
5 \\
-3\end{array}$ & $\begin{array}{r}8 \\
5 \\
-3\end{array}$ & $\begin{array}{r}0 \\
+3 \\
+3\end{array}$ & $\begin{array}{r}0 \\
+1 \\
+1\end{array}$ & $\begin{array}{r}6.0 \\
10.5 \\
+4.5\end{array}$ & $\begin{array}{r}6.4 \\
17.9 \\
+11.5\end{array}$ \\
\hline 26. H.B. & $\underset{\Delta}{\mathrm{AF}}$ & $\begin{array}{r}38 \\
58 \\
+20\end{array}$ & $\begin{array}{r}41 \\
75 \\
+34\end{array}$ & $\begin{array}{r}135 \\
93 \\
-42\end{array}$ & $\begin{array}{r}151 \\
100 \\
-51\end{array}$ & $\begin{array}{r}13 \\
7 \\
-6\end{array}$ & $\begin{array}{r}13 \\
11 \\
-2\end{array}$ & $\begin{array}{r}12 \\
8 \\
-4\end{array}$ & $\begin{array}{r}14 \\
13 \\
-1\end{array}$ & $\begin{array}{l}-1 \\
+1 \\
+2\end{array}$ & $\begin{array}{l}+1 \\
+2 \\
+1\end{array}$ & $\begin{array}{r}4.9 \\
7.5 \\
+2.6\end{array}$ & $\begin{array}{r}8.0 \\
11.9 \\
+3.9\end{array}$ \\
\hline
\end{tabular}

* PLVD $=$ mean left ventricular diastolic pressure; PLVED = left ventricular end-diastolic pressure; $\dot{W}_{L V}=$ left ventricular work; other abbreviations as in Table II. 
pressures occurred after conversion with slight stroke volume increases but without major rate changes. In two without other evidence of heart disease (No. 25 and 26) rather striking decreases in these pressures occurred with clear rate decreases and stroke volume increases. No increase of left ventricular end-diastolic over meandiastolic pressure (PLVED - P PVD) was observed. Stroke work changes paralleled stroke volume changes and were similarly unrelated to changes in mean- or end-diastolic left ventricular pressures. Indicator dye injected into the left ventricle was not detected by continuous sampling in the left atrium in these five patients.

\section{Discussion}

Conversion from atrial fibrillation to normal sinus rhythm was clearly associated with a significant increase in cardiac output in those patients in whom a considerable decrease in ventricular rate occurred. In those patients in whom a significant rate decrease did not accompany conversion, however, the output changes observed at 1 hour were similar in magnitude to those that occurred in patients subjected to the same protocol who did not convert and, furthermore, were similar to those occurring in other patients with heart disease in rest-rerest studies. Output measurements made at longer intervals after conversion in five patients were not regularly greater than at 1 hour. We must conclude that atrial fibrillation had had a deleterious effect on the cardiac outputs of only those patients who had had rapid ventricular rates, as suggested years ago by Lewis (12) and Blumgart (13).

These data were somewhat surprising, however, both from clinical and from physiologic points of view. As physicians, we have all seen quite ill patients who seemed to be improved after conversion. We have also observed acute decreases in cardiac output when atrial arrhythmias developed during cardiac catheterization, as have others (14). That atrial systole plays a role in ventricular filling has clearly been established in animal preparations with induced atrial fibrillation. Skinner, Mitchell, Wallace, and Sarnoff (15) showed that in dogs the absence of atrial systole had two consequences at a given heart rate, namely, decreased ventricular filling and, for a given input into the left ventricle, higher mean left atrial pressure. If the vagi had been sectioned, they noted decreased aortic pressure and cardiac output. If, however, baroreceptor activity was intact, aortic pressure and flow tended to return to control levels after the onset of atrial fibrillation.

Obviously, even among patients with heart disease, little or no increase in cardiac output after conversion would be anticipated in those whose outputs were normal in atrial fibrillation. We had, however, expected an increase in output after conversion in those patients in whom it had been low, on the assumption that the absence of atrial systole was a major factor contributing to their low outputs. Why restoration of atrial systole without a rate decrease did not result in an increase in cardiac output is not clear. The "a" waves that appeared were surprisingly small and were not accompanied by elevation of enddiastolic over mean left ventricular diastolic pressure. In contrast to the findings in dogs without heart disease, the transport function of the atria in these patients seemed to be small. Perhaps this may be attributable in part to an atrial myocardial abnormality that was associated with the development and maintenance of atrial fibrillation. That atrial disease was present in the majority is also suggested by the high incidence of recurrence of fibrillation despite chronic quinidine and procaine amide therapy. Perhaps also this small volume augmentation was in part due to decreased atrial contractility induced by homeostatic autonomic reflexes (16).

Even a small volume augmentation, however, might have been expected to have a salutary effect on stroke output of patients with the lowest cardiac outputs, but the patients with the lowest outputs during chronic atrial fibrillation did not regularly have the greatest percentage increases after conversion. These observations suggest that factors other than the presence or absence of atrial systole were of major importance in setting the level of cardiac output in these patients. By usual clinical criteria, we could not establish a correlation between the severity of the heart disease and the level of cardiac output before or after conversion at rest or during exercise. We are, therefore, unable to define the factors responsible for the low outputs of these patients. 
The question of the effect of atrial fibrillation on left atrial pressure is of definite clinical importance, since this pressure reflects the level of pulmonary venous pressure and hence is related to pulmonary congestion. In the animal preparation with intact baroreceptor activity, mean atrial or filling pressure at a fixed heart rate tends to be higher for any given stroke volume after production of atrial fibrillation even though flow and blood pressure have returned to control levels (15). This was indeed the case in our two patients with atrial fibrillation as the only sign of possible heart disease (No. 25 and 26) in whom clear decreases in both end- and mean-diastolic left ventricular pressures and in left atrial pressures occurred after conversion in association with definite increases in stroke volume. A sharper $\mathrm{x}$-descent occurred in the atrial tracings, suggesting earlier mitral and tricuspid valve closures (15. 17) ; but mitral regurgitation, a possible cause of increased left atrial pressure, could not be detected by an indicator technique in these two patients during atrial fibrillation.

By contrast, in three other patients, one with hypertensive heart disease and two with mitral stenosis, increases in both end- and mean-diastolic pressures occurred without major stroke volume or output changes; increases in left atrial pressure also occurred. An increase in wedge pressure following conversion also was noted by Broch and Müller ( 7 ) in patients with mitral stenosis. The occurrence of this phenomenon in their quinidine-converted patients and the decrease in wedge pressure in two patients with atrial fibrillation only in our series clearly exclude a "stiffening" of the left heart due to any electrically induced. increased catecholamine concentration (18). Although we are unable to explain the phenomenon. we suggest that any improvement in symptoms of pulmonary congestion in patients with mitral stenosis after conversion to sinus rhythm is most likely related to a decreased rate and a longer diastolic filling period, since both conversion per se and an output increase would tend to increase left atrial pressure.

The patients in this and in the majority of previously reported studies have been well enough. despite the presence of serious heart disease, to undergo cardiac catheterization on at least two separate occasions and were studied only supine at moderate exercise. They may not be quantitatively comparable, therefore, to patients who have more severe degrees of cardiac malfunction or to patients who perform more marked activity in the erect position (19). In the upright patient venous return is decreased; ventricular filling and hence stroke volume may be more dependent on the presence or absence of atrial systole.

These studies suggest that chronic atrial fibrillation in these supine and moderately exercising patients with heart disease was of only slight importance in determining the level of cardiac output if the ventricular rate was controlled: The small "a" waves suggesting only a slight volume angmentation attributable to the restored atrial systole, in contrast to the findings in the experimental animal, suggest to us that intrinsic atrial myocardial disease had attenuated atrial function or that extracardiac factors had decreased the contractility of the atria.

\section{Summary}

1) Hemodynamic measurements have been made at rest and during exercise in 20 patients with heart disease before and after attempts to convert atrial arrhythmia to sinus rhythm by transthoracic direct-current shock.

2) Conversion to normal sinus rhythm had little effect on cardiac output unless a rapid ventricular rate was lowered after conversion to sinus rhythm.

3) The transport function of the atria in these patients with heart disease seemed to be of little importance in determining their cardiac outputs.

\section{References}

1. Wade, G., L. Werkö, H. Eliasch, A. Gidlund, and H. Lagerlöf. The haemodynamic basis of the symptoms and signs in mitral valvular disease. Quart. J. Med. 1952, 21, 361.

2. Ferrer, M. I., R. M. Harvey, L. Werkö, D. T. Dresdale, A. Cournand, and D. W. Richards, Jr. Some effects of quinidine sulfate on the heart and circulation of man. Amer. Heart J. 1948, 36, 816.

3. Kory, R. C., and G. R. Meneely. Cardiac output in auricular fibrillation with observations on the effects of conversion to normal sinus rhythm (abstract). J. clin. Invest. 1951, 30, 653.

4. Hecht, H. H., W. J. Osher, and A. J. Samuels. Cardiovascular adjustments in subjects with organic heart disease before and after conversion 
of atrial fibrillation to normal sinus rhythm (abstract). J. clin. Invest. 1951, 30, 647.

5. Hansen, W. R., R. L. McClendon, and J. M. Kinsman. Auricular fibrillation. Hemodynamic studies before and after conversion with quinidine. Amer. Heart J. 1952, 44, 499.

6. Storstein, O., and $\mathrm{H}$. Tveten. The hemodynamic effect of restoring normal sinus rhythm in patients with auricular fibrillation. Scand. J. clin. Lab. Invest. 1955, 7, 167.

7. Broch, O. J., and O. Müller. Haemodynamic studies during auricular fibrillation and after restoration of sinus rhythm. Brit. Heart J. 1957, 19, 222.

8. Lown, B., J. Neuman, R. Amarasingham, and B. V. Berkovits. Comparison of alternating current with direct-current electroshock across the closed chest. Amer. J. Cardiol. 1962, 10, 223.

9. Lown, B.; R. Amarasingham, J. Neuman, and B. Berkovits. The use of synchronized direct-current countershock in the treatment of cardiac arrhythmias. J. clin. Invest. 1962, 41, 1381.

10. Graettinger, J. S., J. J. Muenster, L. A. Selverstone, and J. A. Campbell. A correlation of clinical and hemodynamic studies in patients with hyperthyroidism with and without congestive heart failure. J. clin. Invest. 1959, 38, 1316.

11. Graettinger, J. S., J. J. Muenster, C. S. Checchia, R. L. Grissom, and J. A. Campbell. A correlation of clinical and hemodynamic studies in patients with hypothyroidism. J. clin. Invest. 1958, 37,502 .

12. Lewis, T. Fibrillation of the auricles: its effects upon the circulation. J. exp. Med. 1912, 16, 395.
13. Blumgart, $H$. The reaction to exercise of the heart affected by auricular fibrillation. Heart 1924, $11,49$.

14. Braunwald, E., and C. J. Frahm. Studies on Starling's law of the heart. IV. Observations on the hemodynamic functions of the left atrium in man. Circulation 1961, 24, 633.

15. Skinner, N. S., Jr., J. H. Mitchell, A. G. Wallace, and S. J. Sarnoff. Hemodynamic consequences of atrial fibrillation at constant ventricular rates. Amer. J. Med. 1964, 36, 342.

16. Mitchell, J. H., J. P. Gilmore, and S. J. Sarnoff. The transport function of the atrium: factors influencing the relation between mean left atrial pressure and left ventricular end diastolic pressure. Amer. J. Cardiol. 1962, 9, 237.

17. Ankeney, J. L., A. P. Fishman, and H. W. Fritts, Jr. An analysis of normal and abnormal left atrial pressure pulse in man. Circulat. Res. 1956, 4, 95.

18. Hardy, J. D., T. Carter, and M. D. Turner. Catechol amine metabolism: peripheral plasma levels of epinephrine (E) and norepinephrine (NE) during laparotomy under different types of anesthesia in dogs, during operation in man (including adrenal vein sampling), and before and following resection of a pheochromocytoma associated with von Recklinghausen's neurofibromatosis. Ann. Surg. 1959, 150, 666.

19. Reeves, J. T., R. F. Grover, S. G. Blount, Jr., and G. F. Filley. Cardiac output response to standing and treadmill walking. J. appl. Physiol. 1961, $16,283$. 